\title{
The Earth Lab 5s (ETL3D/5s) seismic sensor. Design and test
}

\author{
Gioacchino Fertitta ${ }^{1}$, Antonio Costanza ${ }^{1}$, Giuseppe D’anna ${ }^{*, 1}$, Domenico Patanè ${ }^{2}$ \\ (1) Istituto Nazionale di Geofisica e Vulcanologia, Osservatorio Nazionale Terremoti, Rome, Italy \\ (2) Istituto Nazionale di Geofisica e Vulcanologia, Osservatorio Etneo, Sezione di Catania, Italy
}

Article history: received July 12, 2018; accepted November 13, 2019

\begin{abstract}
This work presents a new low cost and low power consumption wide-band (5s) three-component seismic sensor, named ETL3D/5s. The sensor is suitable for seismic regional monitoring (local and regional earthquakes), HVSR measurements, seismic microzonation studies and Structural Health Monitoring (SHM) of civil structures. ETL3D/5s includes a set of three $4.5 \mathrm{~Hz}$ geophones and an electronic circuit that increases the geophone's natural period. The sensor exhibits a period of $5 \mathrm{~s}$ and a power consumption as low as $75 \mathrm{~mW}$. Changes in ambient temperature have a small effect on the frequency response because a temperature compensation system is also implemented. A small and sturdy cylindrical housing contains the electronic boards and geophones. The housing design was supported by a modal FEM analysis, in order not to affect the frequency response. The chosen materials and parts guarantee protection against atmospheric agents and watertightness (IP68 degree). The sensor noise model, partially confirmed by a field test, predicts a power spectral density of $10(\mathrm{~nm} / \mathrm{s}) / \sqrt{\mathrm{Hz}}$ at $1 \mathrm{~Hz}$.
\end{abstract}

Keywords: Seismic sensor; Lippman method; Low cost seismology; Induced seismicity; Building monitoring; Urban Seismic Observatory; FEM modal analysis.

\section{Introduction}

Seismic sensors are classified according to what form of ground motion they measure, namely ground velocity (geophones) or ground acceleration (accelerometers). Each sensor type has different advantages in terms of amplitude range, frequency range, reliability and cost.

Ground motion detection is important in a wide range of technical-scientific fields. In physics, advanced experiments can require precise positioning of components. Civil engineers need to know the characteristics of ground motion to better design large-scale structures. The study of ground motion produced by natural phenomena or human activities help geologists to understand the Earth structure and its dynamic [Weber, 2009]. Clearly, each application requires instruments with different specifications. In particular, in order to start a new design, it is necessary to know the range of amplitudes and frequencies that must be measured. In the history of INGV (Istituto Nazionale di Geofisica e Vulcanologia) (ING until 2000), it is possible to remember only a few cases of design and construction of seismometers. In 1939, 11 Wiechert seismometers were made at the ING laboratories [Calcara, 2004]. In addition, in 1939 a single prototype of a vertical seismograph with a $20 \mathrm{~s}$ period was designed and built [Pannocchia, 1939]. In the seventies, several long-period sensors, similar to Wilip Galitzin seismometers, were 


\section{Gioacchino Fertitta et al.}

manufactured at the ING laboratories. Recently, an electronic design was carried out to extend a Lippmann style seismometer’s dynamic range by using a non-linear feedback circuit [Romeo and Spinelli, 2013].

The main focus of this work is to present the new low cost and low power Earth Lab 5s sensor (ETL3D/5s), a portable wide-band (5s) three-component seismic sensor, designed and assembled at the INGV OBS and Earth Lab of Gibilmanna, Italy. This sensor, primarily designed for monitoring local and regional earthquakes, is also suitable for structural engineering applications and ambient vibration measurements. In the latter case, the seismic sensors must have stability and natural frequency nearby the lowest frequency of interest [e.g. Bard et al., 2008]. Therefore, the $5 \mathrm{~s}$ sensor can be well appropriate for these purposes and for microzonation studies that utilize the H/V ratio technique, thanks also to its low weight and easy transportability. Conversely, the broadband seismometers, with natural period higher than $20 \mathrm{~s}$, are not recommended because they can require a long stabilization time.

In the following, the paper describes the basic mechanical and electronic design steps; the final sections report the technical specifications and field tests.

\begin{tabular}{ccc} 
Parameter & Value & Unit \\
\hline Open circuit damping & $0.56 \pm 5 \%$ & - \\
\hline Natural frequency & 4.5 & $\mathrm{~Hz}$ \\
\hline Standard coil resistance & $375 \pm 5 \%$ & $\Omega$ \\
\hline Open-circuit sensitivity & $28.8 \pm 5 \%$ & $\mathrm{~V} \cdot \mathrm{s} / \mathrm{m}$ \\
\hline Moving mass & 0.0111 & $\mathrm{Kg}$ \\
\hline
\end{tabular}

Table 1. HGS HG-6 coil B specifications.

\section{Mechanical design}

Overall housing structure is made of AISI 316 stainless steel. Each mechanical part and electronic component of the seismometer was 3D modeled. In order to verify the operating spaces and to avoid volume or electromagnetic interferences, an assembly 3D model was created, comprising all the mechanical parts as well as the electronic boards. Figure 1a shows the 3D assembly model of the ETL3D/5s seismic sensor; in Figure $1 \mathrm{~b}$ the same 3D model, without the external cylinder, shows the internal components. In order to guarantee water tightness, appropriate o-rings were installed at the base-cylinder, lid-cylinder (Figure 1b) and connector-lid interfaces. For the same reason, sealing washers (steel-rubber) were put between the closing screws (M4) and the lid. Thanks to these solutions, the seismometer meets the requirements of IP68 standard.

The maximum roughness allowed for external surfaces is $0.8 \mu \mathrm{m}$. This feature increases the resistance to atmospheric agents and facilitates the cleaning, which is necessary after digging up the seismometer. On the bottom side of the base plate, three adjustable feet are located $120^{\circ}$ apart (Figure 1a-c).

Three HGS HG-6 B-Coil geophones (see Table 1) are constrained in three orthogonal slots on the top side of the base plate (Figure 1). It should be recalled that geophone specifications always include the natural and the spurious frequency. The spring mass resonance of the geophone along its working axis is associated with the natural frequency; instead, the spurious frequency is associated with a resonance perpendicular to its normal working axis, resulting from a combination of multiple modes of movement [Faber and Maxwell, 1997]. At the spurious frequency, the transfer function shows a change of phase and a strong variation in sensitivity [Faber and Maxwell, 1997]. The HG-6 B-coil geophones have a natural frequency of $4.5 \mathrm{~Hz}$ and a spurious frequency of $140 \mathrm{~Hz}$.

During the housing modeling phase, in order to avoid an activation of geophone resonant modes, a modal FEM (Finite Element Method) analysis [Kythe and Wei, 2004; Ramamurty, 2010; Chandrupatla, 2004] was carried out. It is noteworthy that the obtained housing has resonance frequencies higher than the geophone spurious frequency $(140 \mathrm{~Hz})$. In addition to the housing parts, also the amplifier board was considered, because it constitutes, together with the two support spacers, a vibrating system of considerable importance in this analysis (as we will see later). Figure 1c shows the set of analyzed parts. 
The FEM analysis was realized in NX Nastran, integrated in Solid Edge, using a total of 142.432 tetrahedral elements and 233.250 nodes. This level of discretization was chosen after a convergence analysis that allowed the authors to assess the modal analysis results when decreasing the average size of the element [Cammalleri and Costanza, 2016; Costanza et al., 2017]. Table 2 reports the simulated interconnection constraints between the various connected components. The first ten natural frequencies of the assembly (components 1-9) are finally reported in Table 3; it is clear that the natural modes occur at frequencies sufficiently higher than the spurious frequency $(140 \mathrm{~Hz})$. Figure 2 shows the natural mode shapes 1-4 of the assembly model, without cylinder to show internal components (the cylinder was removed after analysis). It is noteworthy that in modal analysis, the eigenvectors and therefore the mode shapes, are determined at less than a scale factor.
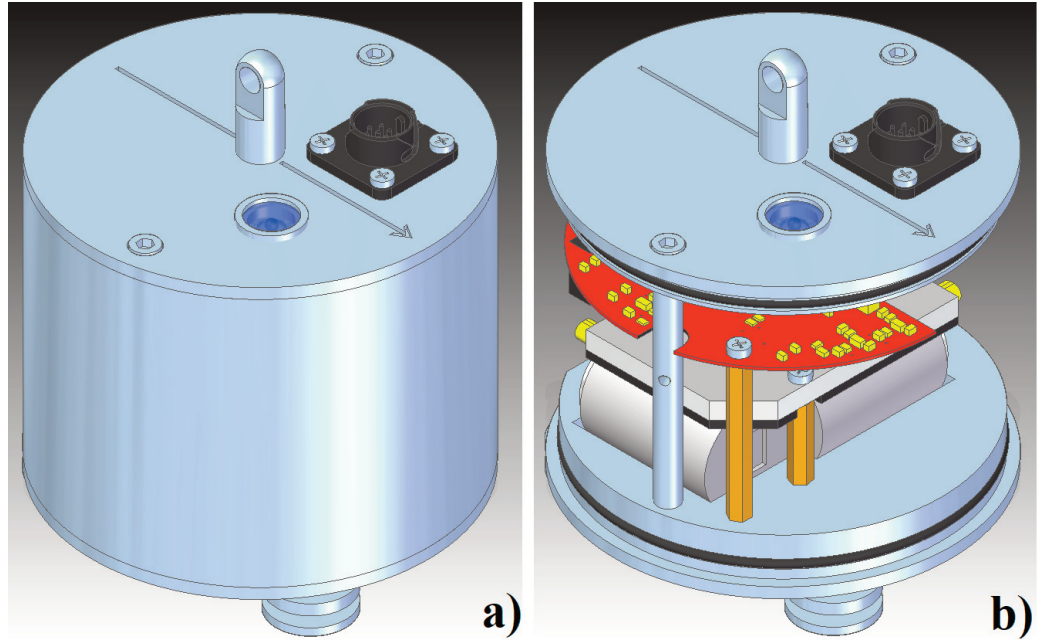

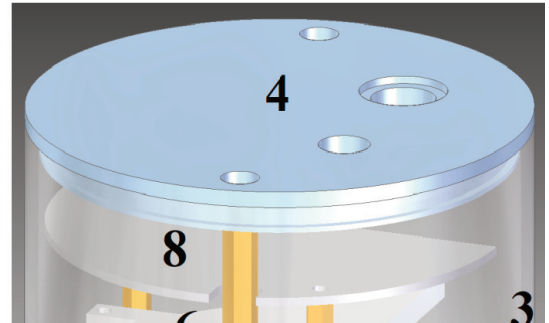

b)

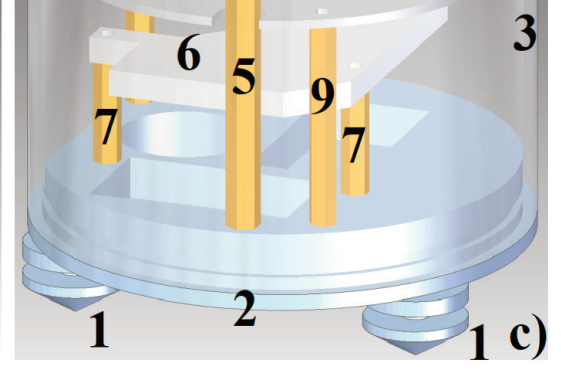

Figure 1.3D assembly model of ETL3D/5s seismic sensor; a) completely closed; b) without cylinder to show internal components; c) with a cylinder in transparency to allow the identification of internal elements used in the modal FEM analysis. 1: adjustable feet, 2: base, 3: cylinder, 4: lid, 5: spacers between lid and base, 6: locking plate for horizontal geophones, 7: spacers for locking plate, 8: board, 9: spacers for board.

\begin{tabular}{cccc} 
Connected components & Constraint \\
Adjustable feet (AISI 316 steel) & Base (AISI 316 steel) & Contact without penetration & Notes \\
\hline Base (AISI 316 steel) & Cylinder (AISI 316 steel) & Contact without penetration & Contact without penetration \\
\hline Cylinder (AISI 316 steel) & Lid (AISI 316 steel) & Threaded connection & Axial preload of 100 N \\
\hline Spacer 5 (AISI 316 steel) & Base (AISI 316 steel) & Threaded connection & Axial preload of 100 N \\
\hline Spacer 5 (AISI 316 steel) & Lid (AISI 316 steel) & Threaded connection & Axial preload of 10 N \\
\hline Spacer 7 (Brass) & Base (AISI 316 steel) & Threaded connection & Axial preload of 10 N \\
\hline Spacer 7 (Brass) & Locking plate (1060 aluminum) & Threaded connection & Axial preload of 10 N
\end{tabular}

Table 2. Interconnection constraints between the various connected components, used in the modal FEM analysis. 


\section{Gioacchino Fertitta et al.}

\section{Components}

Assembly of components 1-9
Natural frequencies [Hz]
349

426

428

497

606

766

$951 \quad 1018 \quad 1153$

Table 3. First ten natural frequencies of the assembly (components 1-9).

Therefore, in Figure 2 only the amplitude ratios must be considered and not their absolute values. The mode shapes show that the lowest resonance frequencies are due to the board-spacers (elements 8-9 in Figure 1c) and to the locking plate-spacers (elements 6-7 in Figure 1c) subsystems, that are the subsets with less flexural stiffness.
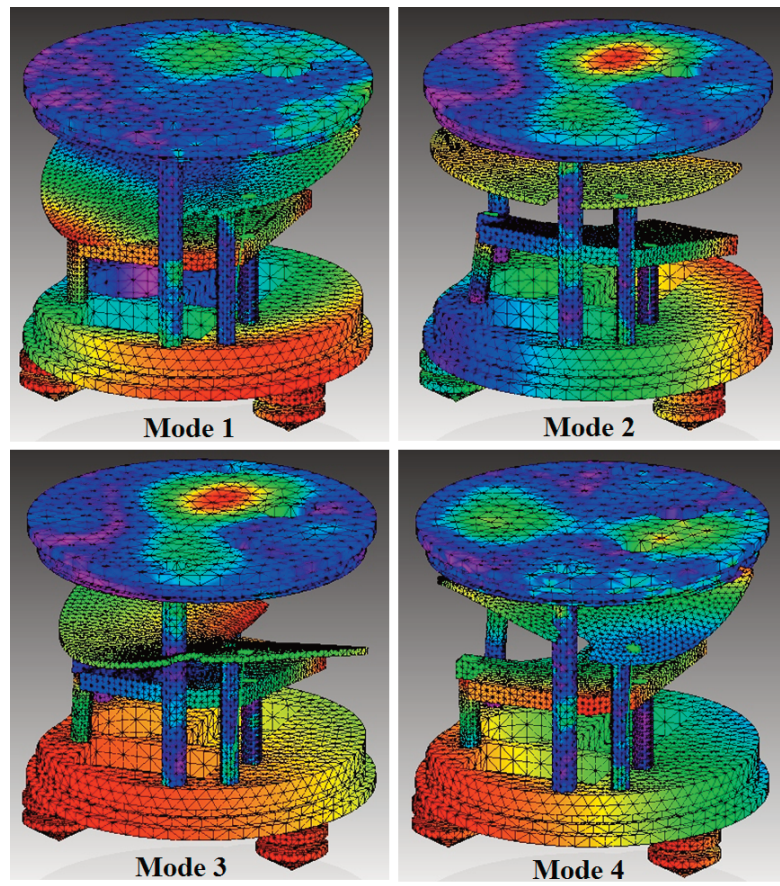

Displacement

Mode 4

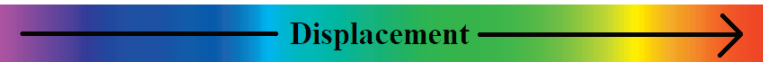

Figure 2. Natural mode shapes 1-4 of the assembly (components 1-9) without cylinder to show internal components (the cylinder was removed after analysis). Warmer colors indicate greater displacements. Image resulting from FEM modal analysis.

\section{The electronics}

\subsection{Architecture}

The electronics includes a set of three geophones and a three-channel amplifier. The amplifier architecture implements the Lippmann's method for increasing geophone bandwidth, the method is widely described in [Romeo and Braun, 2007]. In general, the frequency band lower limit, or lower cutoff frequency, is the most important parameter for seismic sensors, because the seismic spectrum extends to very low frequencies. The upper cutoff is less critical instead, because for most sensors it is well beyond the seismic spectrum upper limit. The Lippmann's method is especially important, because it allows reducing the lower cutoff. For the proposed sensor, a $0.2 \mathrm{~Hz}$ lower cutoff, or $5 \mathrm{~s}$ period, is obtained from the initial $4.5 \mathrm{~Hz}$ of the HG-6 geophone, coil-B type (see Table 1). Chapter I presents examples of surveys and investigations requiring sensors with such a period. 
The proposed circuit could be designed to provide a larger period, but this could be detrimental to the sensor performance. Specifically, the influence of ambient temperature on the transfer function would become stronger. The sensor would rely more on the temperature compensation network, which can only provide a partial correction. Furthermore, as the self-noise density increases with decreasing frequency, the sensor would have a poor dynamic range at very low frequency.

The classical implementation of a Lippmann style velocimeter requires a two-stage amplifier, a negative input resistance amplifier followed by an integrator. In electronics, it is impossible to design an ideal integrator, a low pass active filter is used instead. The cutoff frequency of the filter is set well below the signal frequency, on a logarithmic scale. As a result, the filter response resembles a $20 \mathrm{~dB} /$ decade constant slope curve, that is the shape of the integrator response. The lower the cutoff, the more accurate the integration. Below a certain point, a sensible reduction of the cutoff becomes impractical to achieve without special electronic components, like big size and high cost capacitors. The reason why ideal integrators are not technically possible is that real amplifiers are affected from DC input-errors. Even the smaller DC error can make the circuit saturate, because the integrator has virtually infinite DC gain.

The presented sensor uses an alternative approach to solve the integrator problem. Figure 3 a shows the sensor block-diagram. A feedback network provides a path from the output to the first stage input. As a result, the negative resistance amplifier, integrator and feedback network form a loop. The DC value of the open loop gain is quite large, due to the integrator. This produces a stable operating point, every node in the circuit approaching zero voltage in DC. However, the integrator gain decreases with frequency, and so does the open loop gain. The frequency at which the loop gain amplitude reaches unity defines the lower corner frequency of the sensor. Beyond this value, the loop gain drops below unity and eventually approaches zero. In this condition, the feedback becomes negligible and, for the purpose of the analysis, the circuit can be reduced to the classical version.

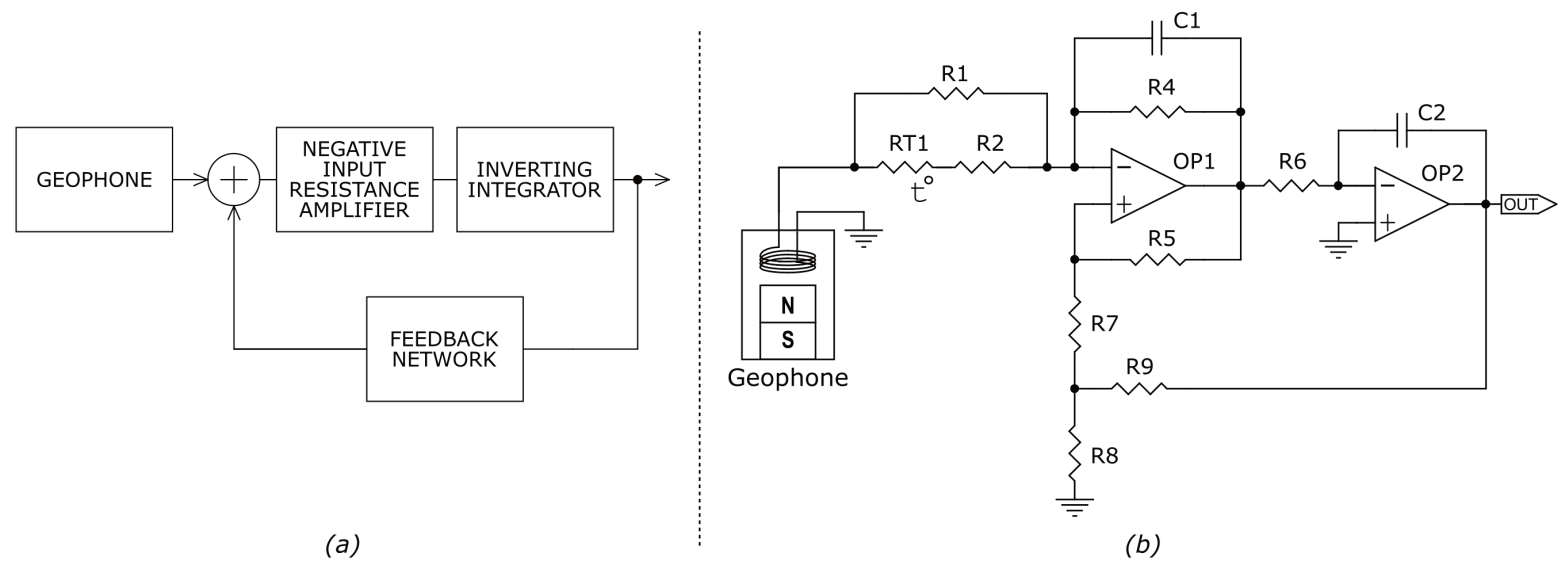

Figure 3. a) Sensor's block diagram; b) ETL3D/5s simplified scheme of the electronic circuit.

\subsection{Circuit implementation}

Figure $3 \mathrm{~b}$ shows a simplified scheme of the circuit. The geophone is to the left of the scheme. The group RT1$\mathrm{R} 1-\mathrm{R} 2$ is a temperature compensation network, which will be discussed later. The operational OP1 with resistors R4-R5-R7 is the negative resistance amplifier. The capacitor C1 is essential for stability, but can be considered an open circuit in the sensor's frequency band. The group OP2-R6-C2 is an inverting integrator, and R8-R9 a resistor divider, feeding the output back to the negative resistance amplifier. Some resistive trimmers, used to adjust the transfer function, are omitted, as well as a third amplification stage, converting the output from single ended to differential.

The expression below shows the sensor's transfer function, arranged in the form prescribed by the SEED (Standard for the Exchange of Earthquake Data) standard [SEED Reference Manual, 2012]. According to the standard, $S_{d}$ is the gain factor, $A_{0}$ the normalization factor, $p_{1}$ and $p_{2}$ are complex poles, defining the lower and upper corner 


\section{Gioacchino Fertitta et al.}

frequency respectively. The normalization frequency is $5.5 \mathrm{~Hz}$. By definition, the gain factor is equal to the sensitivity at the same frequency.

$$
\frac{V_{\text {out }(s)}}{V_{\text {ground }}(s)}=S_{d} A_{0} \frac{s^{2}}{\left(s-p_{1}\right)\left(s-p_{1}^{*}\right)} \cdot \frac{1}{\left(s-p_{2}\right)\left(s-p_{2}^{*}\right)}
$$

The curves in Figure 4 represent the transfer function's magnitude and phase. These curves are calculated using the nominal sensitivity and poles (see Table 4).

The actual transfer function is different from the one shown, due to construction tolerances. However, the resistive trimmers, mentioned above, allows calibrating the transfer function. At the end of the calibration three parameters result verified by measurement, the gain factor $S_{d}$, the real and imaginary part of pole $p_{1}$. As a result, the low frequency portion of the transfer function (below about $10 \mathrm{~Hz}$ ) will be relatively accurate.

The gain-factor calibration was done at the Laboratory of Earthquake Engineering and Dynamic Analysis (L.E.D.A.) of the University of Enna "Kore".

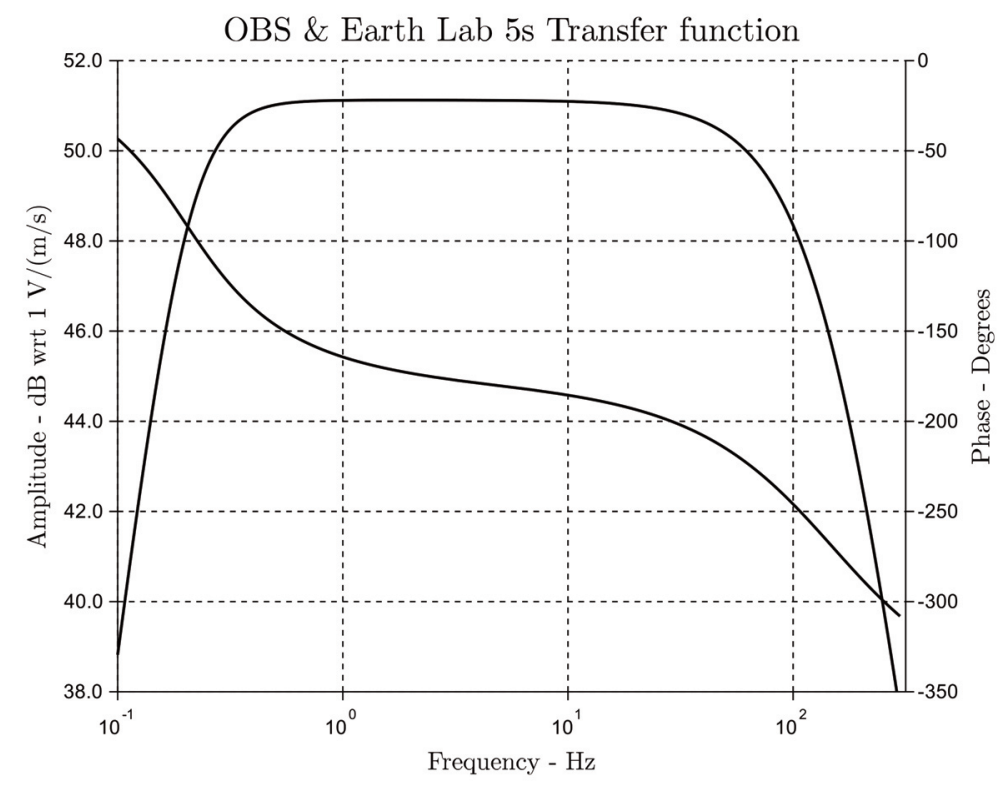

Figure 4. Nominal transfer function (magnitude and phase) of the ETL3D/5s.

The shake table (Figure 5) used for the calibration is the APS420, manufactured by APS. The sensor was excited with a sinusoidal motion at a specific frequency. By adjusting the gain trimmer, it was possible to vary the sensor's gain up to the point where the sensor output is correct, within a certain precision. The sensor was mounted on the table along with a high-end seismic sensor, model Lennartz LE 3D-5s. The latter was suitable for measuring the platform exact velocity, because the sensor's manufacturer performs periodic recalibrations, according to a precise schedule.

The real and imaginary part of $p_{1}$ can be measured by analyzing the sensor's natural response. This can be excited in different ways, for example by applying an electrical impulse of short duration to a convenient node of the circuit. The natural response plot shows a series of exponentially decaying peaks and valleys, whose amplitude and position in time can be used to determine $p_{1}$. Figure 6 shows, for the $\mathrm{z}$ axis, a portion of the natural response, which is similar for the two other components. Table 5 reports the final value of sensitivity, and the real and imaginary parts of $p_{1}$ for each component. Figure 7 shows the low frequency portion of the transfer function after the calibration. The curves are calculated with the values of $S_{d}$ and $p_{1}$ resulting from the calibration. 
The Earth Lab 5s (ETL3D/5s) seismic sensor

General

\begin{tabular}{ll} 
Type & Three-component velocimeter \\
\hline Leveling & Manual through adjustable locking leveling feet and integrated bubble level
\end{tabular}

Sensitivity (nominal value @ $5.5 \mathrm{~Hz}$ ) $360 \mathrm{~V} /(\mathrm{m} / \mathrm{s}$ )

\begin{tabular}{ll} 
& $-0,889+\mathrm{j} 0,889 \mathrm{rad} / \mathrm{s}$ \\
& $-0,889-\mathrm{j} 0,889 \mathrm{rad} / \mathrm{s}$ \\
& $-920+\mathrm{j} 240 \mathrm{rad} / \mathrm{s}$ \\
& $-920-\mathrm{j} 240 \mathrm{rad} / \mathrm{s}$ \\
& double at $0 \mathrm{rad} / \mathrm{s}$ \\
\hline Zeros & $5 \mathrm{~s}$ \\
\hline Period & $12.5 \mathrm{~mm} / \mathrm{s}$ \\
\hline Clip level &
\end{tabular}

Interface

\begin{tabular}{ll} 
Velocity output & $10 \mathrm{~V}$ peak-to peak differ \\
\hline Output voltage swing & $+/-2.5 \mathrm{~V}$ each output \\
\hline Output impedance & $180 \Omega$ each output \\
& $360 \Omega$ in differential mo \\
\hline Connector & 10 poles, Bayonet type \\
& IP68 when fully mated
\end{tabular}

Power

\begin{tabular}{ll} 
Supply voltage & 7 to $15 \mathrm{~V}$ \\
\hline Protection & Reverse-voltage protected \\
\hline Power consumption & $75 \mathrm{~mW}$ at rest $105 \mathrm{~mW}$ maximum \\
\hline Physical & \\
\hline Housing & Stainless steel (AISI 316) \\
\hline Diameter & $97 \mathrm{~mm}$ \\
\hline Height & $117 \mathrm{~mm}$ \\
\hline Weight & $1.7 \mathrm{~kg}$ \\
\hline Parasitic resonances & Above $140 \mathrm{~Hz}$ \\
\hline Environmental & -10 to $50^{\circ} \mathrm{C}$ (by design not tested) \\
\hline Operating temperature & IP68
\end{tabular}

Table 4. ETL/5s specifications. 


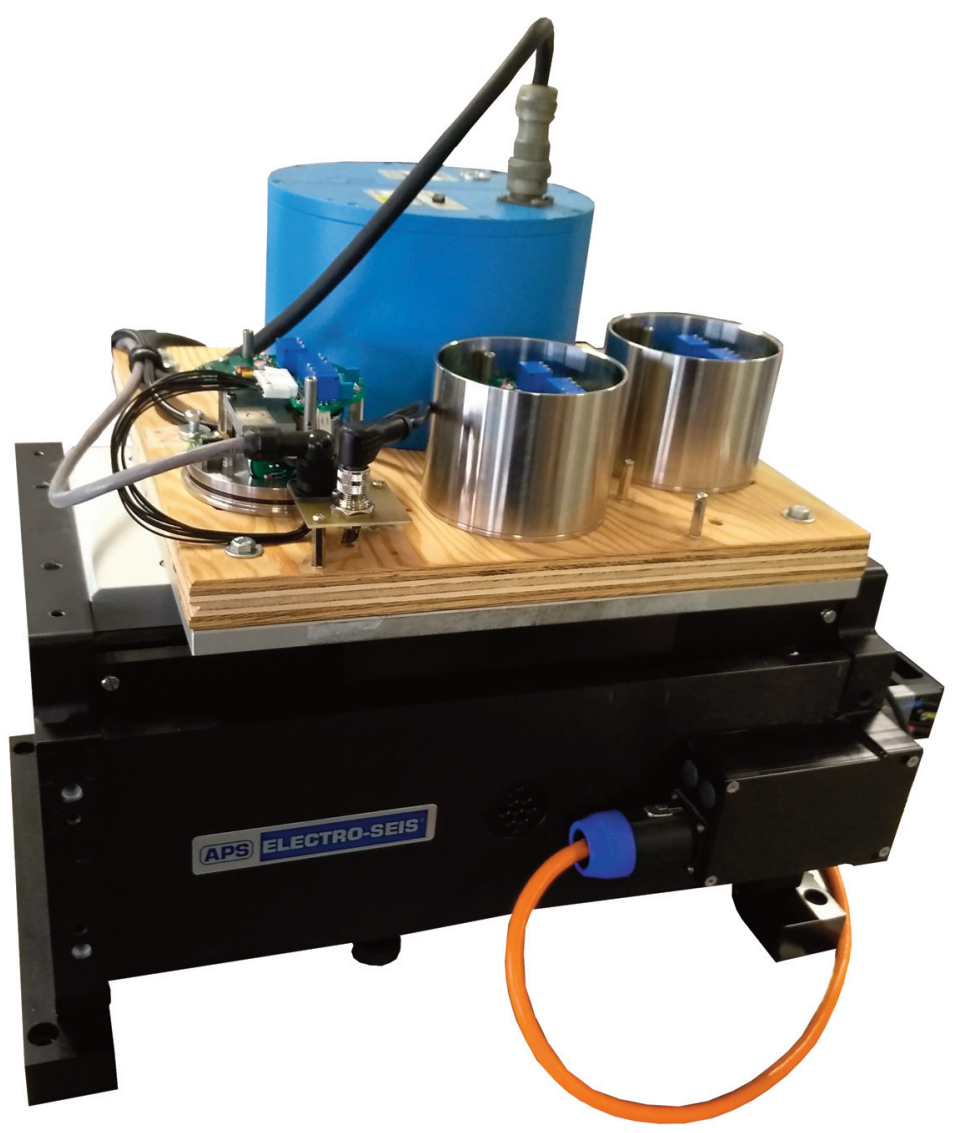

Figure 5. A set of three ETL3D/5 sensors mounted on a shaking table, along with a Lennartz LE 3D 5s (blue case). The latter acts as a reference velocity sensor. The shaking table has been provided by Kore, the University of Enna.

\begin{tabular}{lccc} 
Parameter & Symbol / Expression & Value & Unit \\
Sensitivity Z-axis & GZ & 361 & $\mathrm{~V} /(\mathrm{m} / \mathrm{s})$ \\
\hline Sensitivity EW-axis & GEW & 361 & $\mathrm{~V} /(\mathrm{m} / \mathrm{s})$ \\
\hline Sensitivity NS-axis & GNS & 358 & $\mathrm{~V} /(\mathrm{m} / \mathrm{s})$ \\
\hline Real part of p1 Z-axis & real[p1Z] & 0,863 & $\mathrm{rad} / \mathrm{s}$ \\
\hline Real part of p1 EW-axis & real[p1EW] & 0,869 & $\mathrm{rad} / \mathrm{s}$ \\
\hline Real part of p1 NS-axis & real[p1NS] & 0,888 & $\mathrm{rad} / \mathrm{s}$ \\
\hline Imaginary part of p1 Z-axis & imag[p1Z] & 0,882 & $\mathrm{rad} / \mathrm{s}$ \\
\hline Imaginary part of p1 EW-axis & imag [p1EW] & 0,892 & $\mathrm{rad} / \mathrm{s}$ \\
\hline Imaginary part of p1 NS-axis & imag [p1NS] & 0,889 & $\mathrm{rad} / \mathrm{s}$ \\
\hline
\end{tabular}

Table 5. Measured values of some transfer function parameters. 


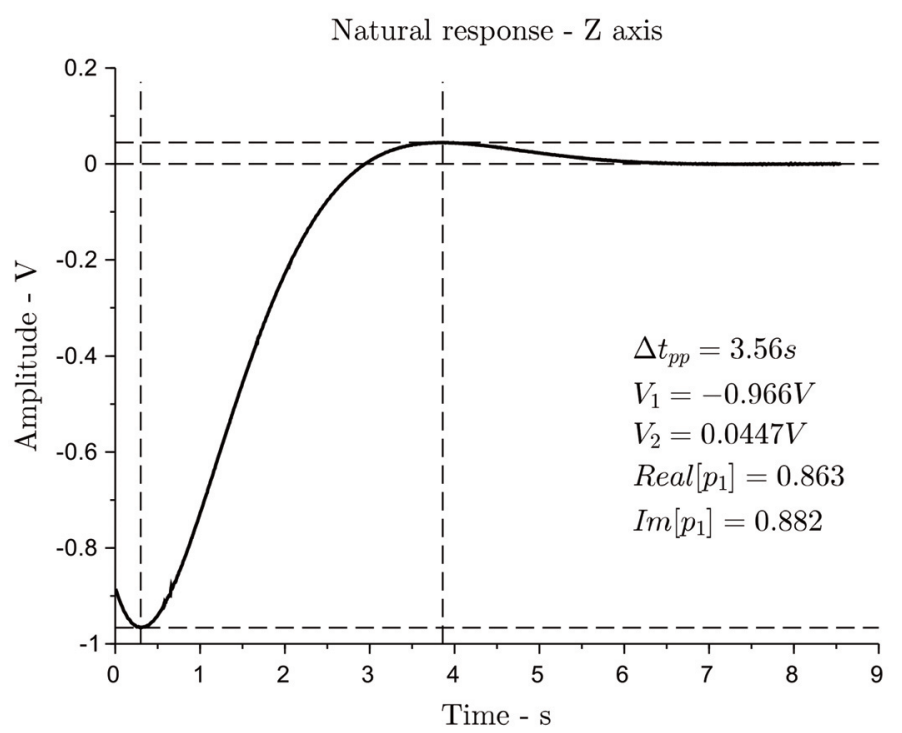

Figure 6. A portion of the natural response for the Z-axis. See table 5 for the final values of sensitivity and the real and imaginary parts of $\mathrm{p} 1$ for the $\mathrm{E} / \mathrm{W}$ and $\mathrm{N} / \mathrm{S}$ axis.

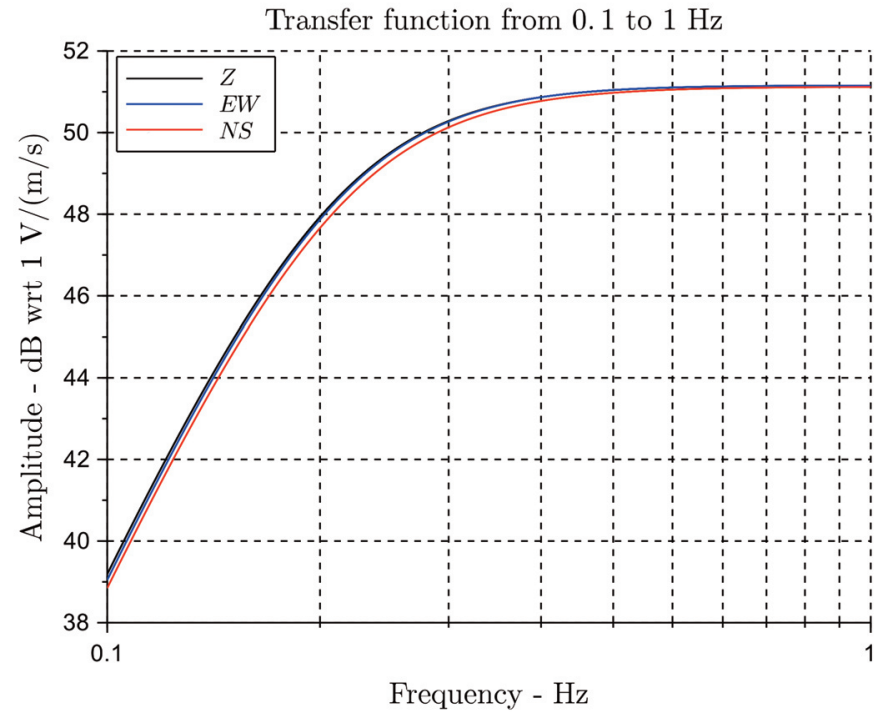

Figure 7. Low-frequency transfer function, magnitude only, all components. These curves are calculated using the values of $\mathrm{G}$ and $\mathrm{p} 1$ resulting from the calibration.

Finally, the temperature compensation network RT1-R1-R2 will be discussed. The transfer function of Lippmann's circuit varies with temperature; the larger the circuit gain, the more pronounced is the variation. The principal cause is the dependence of the geophone coil resistance on temperature [Boulaenko, 2002]. A temperature dependent resistive network is put in series and in thermal contact with each geophone, in order to stabilize the transfer function. The combined resistance, sum of coil and network resistances, has a lower rate of change, or temperature coefficient, than the coil. At room temperature $\left(25^{\circ} \mathrm{C}\right)$, the circuit produces the nominal transfer function. Around this point, the sensitivity to temperature changes is minimum, because the derivative of total resistance with respect to temperature is null. 


\section{Gioacchino Fertitta et al.}

\subsection{Power consumption}

Power consumption is a figure of merit for seismic sensors. The ETL3D/5s consumes $75 \mathrm{~mW}$ at rest. When the sensor is shacked, the maximum power is $105 \mathrm{~mW}$. In terms of autonomy, a $12 \mathrm{~V}-7 \mathrm{Ah}$ battery could supply the sensor for more than 45 days. Other electrical specifications are given in Table 4 .

\section{Self-noise evaluation and field tests}

In order to evaluate the ETL3D/5s self-noise, a test was carried out near the INGV monitoring node of Castelbuono. The location is well suited for this kind of tests, because it shows a low level of ambient noise.

A reference data acquisition system worked in parallel with the main acquisition system. Because the reference system was equipped with a low noise seismometer, Lennartz LE 3D 5/S, its data can be used to evaluate the site's ambient noise. During the recording (Figure 8), whose time length was about 30 minutes, the sensors were placed close to each other, on a hard rock surface.

The start and the end of the time series were excluded from the analysis, in order to get rid of system transients and artificial noise. Power spectral densities were computed with the following algorithm.

First, the time series are divided into overlapping segments. The second step is to calculate the Discrete Fourier Transform (DFT) of each segment, by using the Fast Fourier Transform (FFT) algorithm. The average DFT of all the segments, multiplied by an appropriate factor, becomes a power spectral density, with dimensions $\mathrm{Hz}^{-1 / 2}$.

The final step is the deconvolution, which removes the digitizer and sensor transfer functions, yielding a ground velocity density.

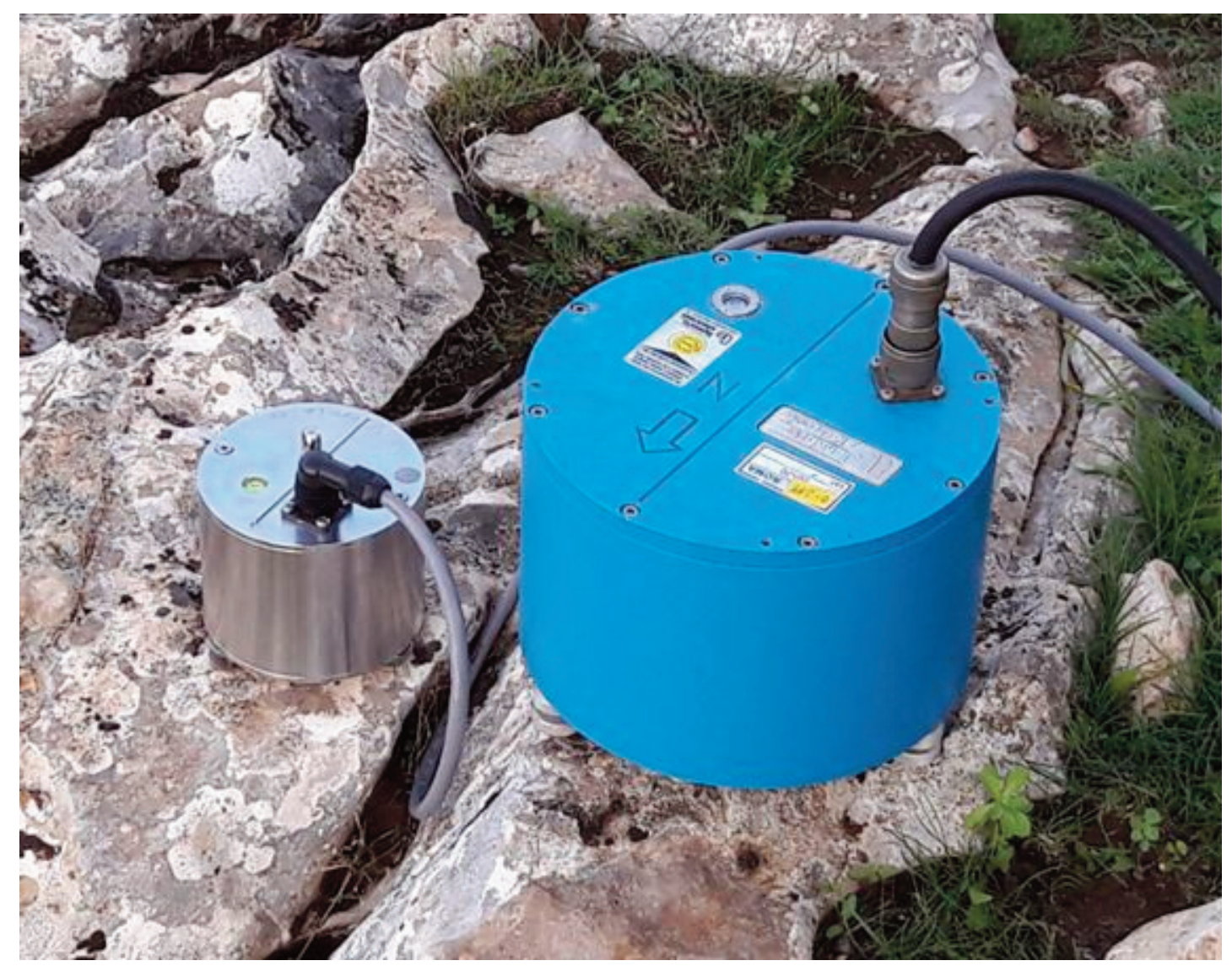

Figure 8. The ETL3D/5s sensor (left) and the reference Lennartz LE-3D 5/S (right) during the test. 
Note that the two sensors have the same band-width, however the LE 3D 5/S implements an extra filtering stage, high pass type, with cutoff frequency of $0.046 \mathrm{~Hz}$, therefore the inverse transfer functions are actually different. Figure 9 shows the spectral densities, grouped by component. Looking at the curves some observations can be made. At frequencies for which LE 3D 5/S spectrum is higher than ETL3D/5s theoretical self-noise, the two sensors gave almost the same density. Where LE 3D 5/S decreases below the theoretical curve, ETL3D/5s gave a higher density than the reference sensor. See for example the interval between 4 and $5 \mathrm{~Hz}$ of component $\mathrm{Z}$. The observations above indicate that the theoretical noise model actually predicts the sensor self-noise level.

For the LE 3D 5/S, the instrument manual shows that the self-noise level is well below those measured in this test [Lennartz, 2016]. Therefore, the LE 3D 5/S density represents the ambient noise while the ETL3D/5s curve shows the sum of ambient and self-noise. For the ETL3D /5s, wherever the ambient noise decreases below the selfnoise level, self-noise becomes dominant. Although the recording was quite short, the theoretical model for sensor's self-noise was partially verified.

Furthermore, in order to evaluate the performance and sensitivity, the ETL3D/5s was tested in a monitoring node of the Catania Urban Seismic Observatory (OSU-CT) [Patanè et al., 2018], characterized by a medium-high level of ambient noise. The OSU-CT has been conceived as a high-density grid of low-cost seismic stations, equipped with Micro Electro-Mechanical Systems (MEMS) accelerometric sensors, installed in the historical center of the city. Moreover, for the purposes of Structural Health Monitoring (SHM) of some selected edifices, the integration with the ETL3D/5s has been planned.

For this test, a reference data acquisition system equipped with a low noise seismometer Lennartz LE 3D/20s works in parallel with the OSU-CT acquisition system. The LE-3D/20s was connected to a Nanometrics Taurus recorder, while the ETL3D/5s was connected to a high-resolution 24 bit digitizer, still under development, specifically designed for the OSU-CT network. Figure 10 shows the comparison of seismic waveforms recorded by the two sensors, both for a local Etna earthquake (20180619, 03:44:00) of Ml 2.5, located about $16 \mathrm{~km}$ away from Catania, and for a Greek event (20180625, 07:14:47) of Ml 5.3, located about $500 \mathrm{~km}$ away from Catania. Furthermore, for each event, the comparison of the spectral content for the P and S-phases is shown. It is noteworthy that the spectral shapes are comparable up to about $20 \mathrm{~Hz}$. Conversely at higher frequencies, signals acquired by the LE-3D/20s has a flatter amplitude-response to velocity, due to a filter used by the Nanometrics Taurus data-logger.

\section{Discussion and conclusion}

The main purpose of this work is to inform the scientific community of the new low cost, low power and low weight portable seismic sensor named ETL3D/5s, designed and assembled at INGV OBS and Earth Lab of Gibilmanna, Italy. The final cost of the sensor, including an estimate for the assembly and calibration work, is of about 2000 Euros. However, a significant margin for cost reduction could come from scale manufacturing and process engineering. It is certainly natural to wonder why not to design a broadband sensor. The answer is that a broadband sensor is much more expensive than the sensor presented here and therefore would make a seismic or structural monitoring overly expensive. Using broadband sensors for seismic noise measurements or local micro-earthquakes recording does not provide more information. In addition, the presented sensor is appropriate for monitoring local and regional earthquakes, for engineering purposes (e.g. for the SHM) and ambient vibration analysis. It can also be used for the realization of low-cost microseismic networks in areas where industrial activities may "induce seismicity"; this last purpose became very important in recent years. In fact in 2014, in Italy, precise monitoring guidelines were established for monitoring seismicity, ground deformation and pore pressure in subsurface industrial activities [MiSE, 2014]. For the sensor design, a modal FEM analysis allowed designing a housing with natural frequencies sufficiently higher than geophones spurious frequency. By implementing the Lippmann's method, a $0.2 \mathrm{~Hz}$ cut-off frequency was achieved, starting from $4.5 \mathrm{~Hz}$ geophones.

Several laboratory and field tests were made in order to evaluate the self-noise level and to verify the transfer function. For these tests, a Lennartz LE 3D 5/S and a Lennartz LE 3D 20/S were used as reference sensors. The comparative analysis greatly helped interpret the results. The ETL3D/5s self-noise was evaluated, finding satisfactory results. The theoretical model for the sensor's self-noise was partially confirmed. However, in order to verify the entire noise model, new tests should be made considering also a new site with less ambient noise. 

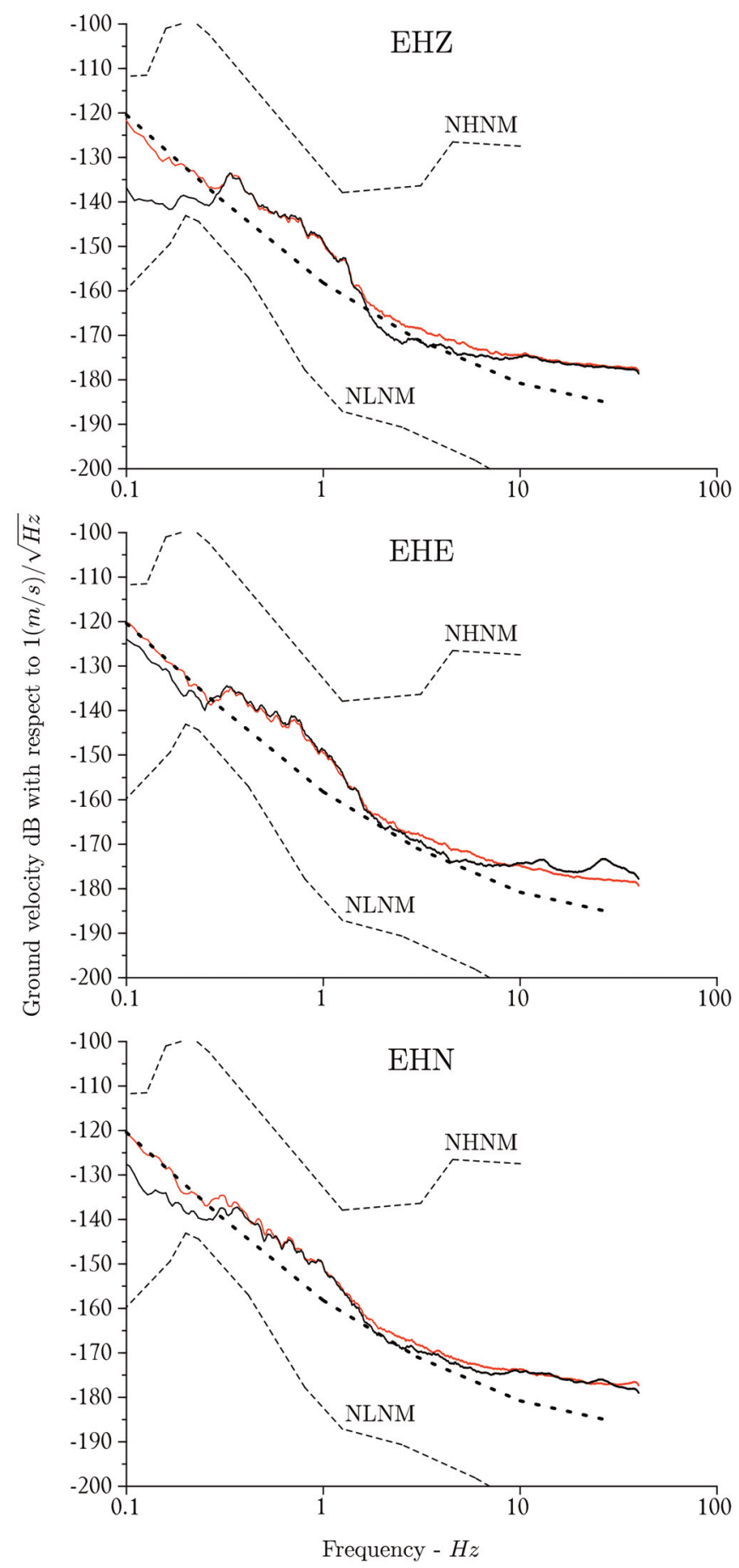

Figure 9. Power spectral densities: LE 3D 5/S (solid red), ETL3D/5s (solid black), Noise models high and low (dashed), ETL3D/5s theoretical self-noise (dotted). 

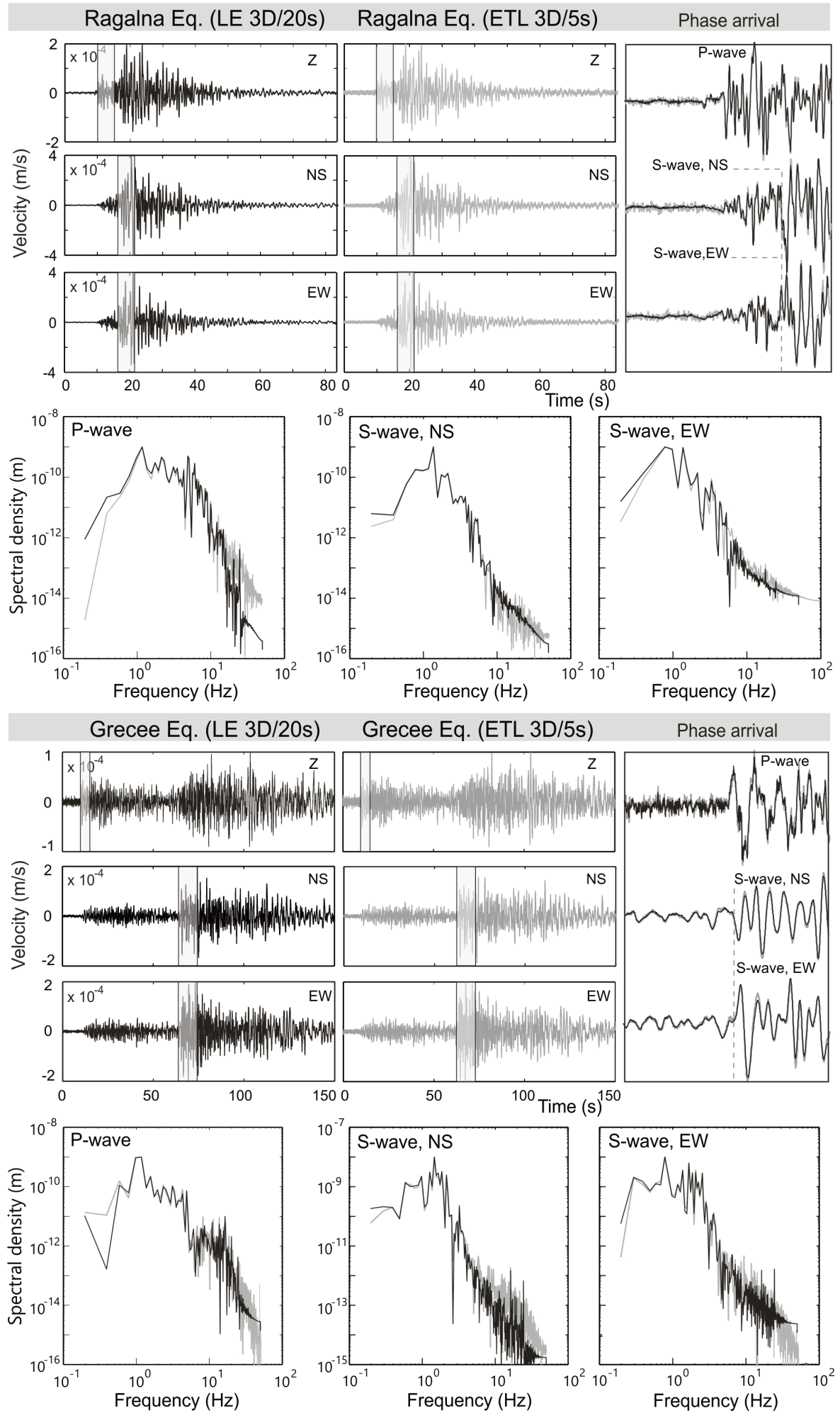

Figure 10. Comparison of seismic waveforms of two earthquakes recorded, at the CTOE test site of OSU-CT, by the ETL3D/5s and Lennartz LE 3D 20/S. For the P and S-phases, the superimposed enlarged waveforms of the two sensors are also shown. For each component (Vertical, North-South and East-West), the comparison of spectral density computed for P and S-phases (grey areas in the seismograms) is also shown. 


\section{Gioacchino Fertitta et al.}

Acknowledgements. We gratefully acknowledge the editors Giovanni Romeo and Gaetano De Luca, whose suggestions improved the manuscript significantly. This work has been supported by the following: the MISE project and the PONARS01_00926 project eWAS “An Early Warning System for Cultural Heritage”. We also thank Giuseppina Tusa for the analysis of seismic signals, and Giacomo Navarra, responsible of the Laboratory of Earthquake Engineering and Dynamic Analysis (L.E.D.A.) of University of Enna "Kore" and its staff for the support to sensor calibration.

\section{References}

Bard P-Y, C. Acerra, G. Alguacil, S. Zacharopoulos et al. (2008). Guidelines for the implementation of the H/V spectral ratio technique on ambient vibrations measurements, processing and interpretation. Bull. Earthq. Engi. 6(4), 1-2

Byrne C.J. (1961). Instrument noise in seismometers. Bull. Seismol. Soc Am.. 51, I, 69-84.

Boulaenko M. E. B., Sc. (2002). Novel Tools for Research and Education in Seismology. Master of Science Thesis. Institute of Solid Earth Physics, University of Bergen.

Calcara G. (2004). Breve profilo dell'istituto nazionale di geofisica (1936 - 1963). Quaderni di Geofisica 36.

Cammalleri M. and A. Costanza (2016). A closed-form solution for natural frequencies of thin-walled cylinders with clamped edges. International Journal of Mechanical Sciences 110, 116-126. doi:10.1016/j.ijmecsci.2016.03.005.

Chandrupatla T. (2004). Finite Element Analysis For Engineering \& Tech. Universities Press (India).

Costanza A., G. D’Anna, A. D’Alessandro, G.Vitale, G. Fertitta, P. Cosenza (2017). Mechanical aspects in a tide gauge station design. Conference paper. Oceans'17 MTS/IEEE Aberdeen, Scotland. DOI:10.1109/OCEANSE.2017.8084642.

Faber K. and P. W. Maxwell (1997). Geophone spurious frequency: what is it and how does it affect seismic data quality? Can. J. Explor. Geophys. 33, 1- 2, 46-54.

Havskov J. and G. Alguacil (2004). Instrumentation in Earthquake Seismology. Springer.

Kythe P. K. and D. Wei (2004). An Introduction to Linear and Nonlinear Finite Element Analysis. Birkhauser.

LE-xD Seismometer Family. Document Number: 990-0073

Ministry of Economic Development (MiSE) (2014). Guidelines for monitoring seismicity, ground deformation and pore pressure in subsurface industrial activities.

Pannocchia G. (1939). Sismografo verticale a 20s di periodo proprio. Pubblicazioni dell'Istituto Nazionale di Geofisica del Consiglio Nazionale delle Ricerche ${ }^{\circ} 11$.

Patanè D. \& Gruppo di Lavoro OSU-CT (2018). La rete accelerometrica urbana nel centro storico della città di Catania (OSU-CT). Pubblicazioni Geologia dell’Ambiente- Supplemento al n. 1/2018, ISSN 1591-5352, 249-257.

Ramamurty G. (2010). Applied Finite Element Analysis. I.K. International Publishing House Pvt. Ltd. Second edition 2010.

Romeo G., F. Pongetti, G. Spinelli (2012). Simulazione di un geofono utilizzando pSPICE. INGV technical note 242, ISSN 2039-7941

Romeo G. and T. Braun (2007). Appunti di sismometria. Quaderni di Geofisica 46.

Romeo G. and G. Spinelli (2013). Extending a Lippmann style seismometer's dynamic range by using a non-linear feedback circuit. Adv. Geosci. 36, 27-30.

SEED Reference Manual. Standard for the Exchange of Earthquake Data. IRIS, Version 2.4 August, 2012.

Weber A. (2009). Design, fabrication, and operation of two broadband force balance seismometers. Louisiana State University Master's Theses.

"CORRESPONDING AUTHOR: Giuseppe D'ANNA,

Istituto Nazionale di Geofisica e Vulcanologia, 